\title{
Damages Recognition on Crates of Beverages by Artificial Neural Networks Trained with Data Obtained from Numerical Simulation
}

\author{
Jörg Zacharias, Christoph Hartmann, and Antonio Delgado \\ Technische Universität München \\ Chair of Fluid Mechanics and Process Automation \\ Weihenstephaner Steig 23, 85350 Freising-Weihenstephan, Germany \\ Joerg.Zacharias@wzw.tum.de
}

\begin{abstract}
A new method to detect damages on crates of beverages is investigated. It is based on a pattern-recognition-system by an artificial neural network (ANN) with a feedforward multilayer-perceptron topology. The sorting criterion is obtained by mechanical vibration analysis which provides characteristic frequency spectra for all possible damage cases and crate models. To support the network training, a large number of numerical data-sets is calculated by finite-element-method (FEM). The combination of artificial neural networks with methods of numerical simulation is a powerful instrument to cover the broad range of possible damages. First results are discussed with respect to the influence of modelling inaccuracies of the finite-element-model and the support of ANN by training-data obtained from numerical simulation.
\end{abstract}

\section{Introduction}

Based on the project-idea to improve the quality requirements on deposit systems and filling lines in the beverage industry [1], a new recognition method is presented in the current contribution. The increasing variety of crate models complicates the sorting as well as added impurities and ageing of the material. Therefore, functional sorting systems are needed for the inspection of returned reusable crates of beverages which are mainly sorted by optical systems in industrial automatic filling lines. The general problem of these lines is the detection of small and hidden damages.

The scientific motivation results from some contributions in aerospace, civil engineering, seismic research and some basic mechanical engineering problems. They describe methods to detect damages and failures in structures using frequency response and transient response data of vibrating mechanical systems ([2], [3], [4]). Also numerical simulation on frequency response methods of different examples as plates, beams, bridges, buildings have been done with good agreement on experimental results ([5], [6]). Further research show the capability of artificial neural networks (ANN) in different topologies to classify some damages of structures which receive data out of different vibration based analysis ([7], [8]). This short review shows that the basic principles have been proven on buildings, aircraft-wings, beams and plates by different authors. The combination of all these principles to get finiteelement (FE) supported ANN trained by numerical simulated data-sets have been 
taken mainly at simple structures like plates, beams and strongly simplified models ([9], [10]), where also some problems to get fitting results with this technique are discussed [11]. This also underlines that there is not an overall solution and each single case needs its special adaptation.

The following contribution will apply the methods to the complex structure of a crate of beverage. The basic idea and the feasibility of the method have been reported in [12]. The development of a pattern-recognition-system for damaged polyethylene crates of beverages is carried out on the principle of mechanical excitation and frequency response measurement. The aim of the system is to take advantage of these reliable mechanical methods and combine them with the quick, adaptive pattern recognition of ANN which allows automatic sorting. Additionally, numerical simulation of the mechanical system provides many information about the system behaviour for the planning of the experimental device and for further use in network training. Typical damages as flaws, deformations or separated components are considered in the current contribution.

\section{Analysis}

In order to meet the principle objectives of the system, the damage detection of polyethylene crates of beverages, based on the pattern recognition of vibration response analysis data is established. This technique is taking advantage of the property of mechanical systems to transmit signals through the whole structure. The vibration response of damaged crates or crates of different kind vary from that of a standard (undamaged) crate. The difference is used as a criterion to select individual crates. The pattern recognition of various response spectra is done by ANN. Since a large number of possible damages occur, the training is carried out with data obtained from experimental analysis and from numerical simulation. Also the data reduction (pre-processing) of the response spectra to an appropriate amount of characteristic input data is described.

In the experimental part of the present project a mechanical excitation is applied to a crate of beverage. Therefore, a customary polyethylene crate is fixed on an electrodynamic vibration facility (see figure 1). Control by a PC-control-system provides a sinusoidal vertical movement of the shaker-piston where the crate is fixed on a central position on a specially constructed expander. The excitation is carried out over a sweep of a frequency range from 50 to $1000 \mathrm{~Hz}$, controlled by an amplitude of constant power with a starting acceleration of $75 \mathrm{~m} / \mathrm{s}^{2}$ which also is the reference for the system calibration. This excitation does not cause any visible destruction or plastic deformation to the crates. The system answer is recorded separately as an acceleration signal over the whole frequency range at different locations on the crate by several acceleration-sensors. Mainly observed for the described results is one control sensor at the expander and one reference sensor on the middle of the handle of the crates. All kind of crates and damages can be processed in this way. In the present case, 20 crates with different, empirically evaluated damages are used to cover a basic range of damages. Flaws, deformations, separated components on sides, handles, compartments and also multiple damages as well as three different models are inspected. 


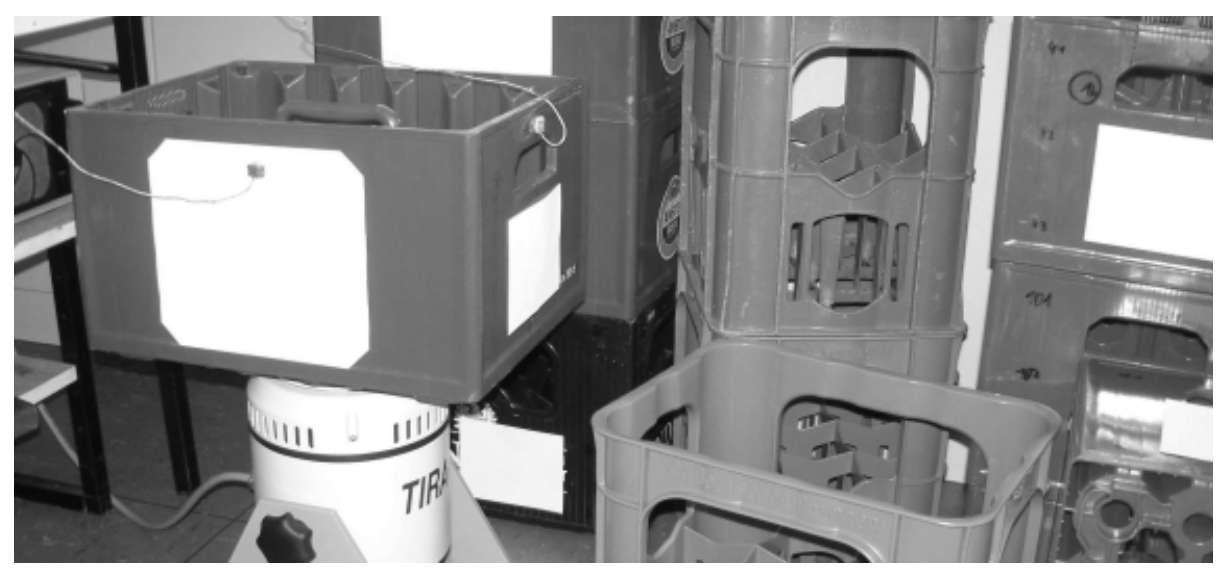

Fig. 1. Vibration system at the laboratory

The vibrational motion can be described mathematically by the general equation of motion (1) which is the basis for the numerical analysis of the system. In a finiteelement model inertia (M), damping (D), stiffness (K) of the model are determined by the geometry and the material properties. The force $f(t)$ defines the excitation, and $u$ represents the displacement vector of all degrees of freedom of the discretized model.

$$
[M]\{\ddot{u}\}+[D]\{\dot{u}\}+[K]\{u\}=\{f\}(t)
$$

From modal analysis it is known, that the displacement vector $\{\mathrm{u}\}$ can be expressed as a series of superposed eigenmodes $\Phi_{i}$ with different amplitudes $\xi_{i}$.

$$
\{u\}=\sum_{i}\left[\Phi_{i}\right] \xi_{i}
$$

The natural frequencies of a structure are the frequencies at which the structure naturally tends to vibrate if it is subjected to a disturbance. The deformed shape of a structure at a specific natural frequency is called its mode shape of vibration. Natural frequencies and mode shapes are functions of the structural properties (e.g. material parameters, geometry). All the modes and frequencies are system immanent. If the excitation frequency is equal to one of the natural frequencies the responseamplitudes become very large. This is called resonance. In the case of enforced motion, the amplitude and the phase of the vibration at a distinct point are recorded. Excitation in the representative frequency range results in associated response frequency spectra which are specific for each observed measurement point as well as for each geometry, including damages, and different material properties.

Based on this knowledge, numerical simulation is carried out in order to provide both, insight into the basic vibration behaviour and frequency response spectra for the training of the ANN. The evaluation of the mode shapes ensures the optimisation of the experiment and can guide through it. In pre-test planning stages standard mode shapes can be used to indicate the best location for the accelerometers and for the position of excitation [13]. Furthermore, there is an enormous need of data-sets for the 
network-training and network-testing, that represent a wide range of crates of equal damages and also a wide variety of possible damages, whose experimental data collection is very costly. These data-sets can be produced in a more efficient way by numerical simulation.

A CAD-data-set is automatically meshed with support of the pre-processor MSC/PATRAN, which leads to a model of more than 1.000.000 degrees of freedom. Two different element types are deployed, 4 node (TET4) and 10 node (TET10) tetrahedral elements [14]. The finite-element-solver MSC/NASTRAN is configured to a frequency response analysis to calculate comparable spectra of the vibration behaviour of the crates, intact as well as damaged. For this purpose some simplifications are done within the configuration. Therefore, usually the first step is the calculation of mode shapes and natural frequencies. No damping is used which leads to results that characterise the basic dynamic behaviour of the structure and indicate how the structure will respond to dynamic loading. To simulate frequencyresponse-spectra another method is applied, which uses the technique of the largemass-principle, where applied forces are used in conjunction with concentrated masses (see [15], [16], [17] for further information). These spectra are validated by comparison with the experimental data and by optimisation of the parameters and the simulation configuration of the system and the solver. The validated numerical database now is applicable for any possible damage case in further simulations. This data can be included into the training-data-set to enhance experimental training-data and cover a broad range of possible damages.

The third part of the system, the pattern recognition by ANN, is done with a multilayer-perceptron (MLP) network. As also reported by [18] the MLP is proved to be an appropriate topology for the desired classification. A network with 10 input nodes and 5 nodes in the hidden layer, all connected in forward direction, is built up in order to classify the signal in 2 output nodes as "intact" or "damaged" (figure 2).

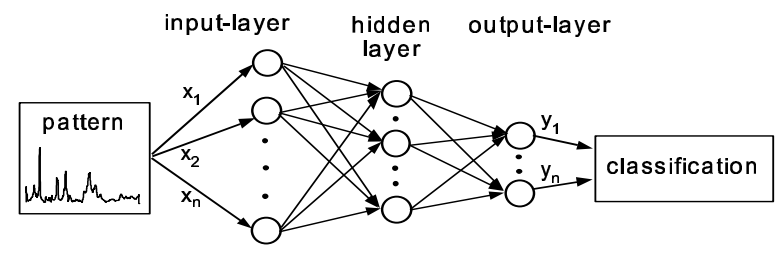

Fig. 2. Multilayer-perceptron network

The initial worths of each input are summed up and weighted in the nodes by

$$
z_{i}=\sum_{j=1}^{n} w_{j i} x_{i}
$$

leading to the activation of each node by the common type of a sigmoid transfer function

$$
S_{i}(\vec{x})=1 /\left(1+\exp \left(-z_{i}\right)\right)
$$


Here $\mathrm{x}_{\mathrm{j}}$ is the activity of the $j$-th input node and $S_{i}(\vec{x})$ is the activity of the $i$-th hidden layer node. This effects a binary output, which leads in the output layer to the prediction of each output node to a " 1 " for true and a " 0 " for false, as also reported by [19]. Starting with a random weight $\mathrm{w}_{\mathrm{ji}}$ of each connection the supervised learning algorithm resilient propagation (RPROP) is used for the network training to adjust the weights of the initial connections to minimise errors between network output and the output target. For this feed-forward network RPROP performs a fast and robust learning algorithm, which adjusts the interconnecting weights by adapting each weight automatically in order to minimise the error function by correcting them because of the gradients algebraic sign ([20], [21]). As ANN are only able to process data in a certain format, data-reduction by splitting and integrating the spectra yields a suitable amount of data for the input nodes. Each input node processes a normalised part of response range and is not dependent on any previous knowledge about the model behaviour. A minimum of non-redundant data-sets is needed for training ( 20 to 50 different cases at the moment). This number depends on the variety of the damages. The implementation of more crate models will cause more training-datasets.

\section{Results and Discussion}

The mode shape analysis gives insight into the preferred state of motion of the mechanical structure. Therefore, it can be evaluated in order to choose sensor positions which provide a high degree of sensitivity. For example, in figure 3, the fifth mode shape is shown. The region around the handles is strongly deformed. This means, that a sensor placed on the handle would record a high amplitude. A damaged handle would be recognised because of a different mode shape at a different frequency.

The experimental results show, concerning the feasibility of the damage recognition method, that different cases of detected spectra show different peaks with different amplitudes and positions depending on the damages. See figure 4 for typical vibration spectra at one reference point on the handle of an intact and two damaged crates.

It can be stated that damages on crates of beverages can be identified by the comparison of vibration spectra. Also the small and hidden damage in the compartment is observable. In detail, the peaks are specific in position and size, especially in two main regions from 120 to $250 \mathrm{~Hz}$ and from about 400 to $850 \mathrm{~Hz}$. This is mainly due to the geometry modifications of every sample. The reproducibility of the spectra measured by the described method is possible in all cases. This is proved by repeated measurements. However, to obtain data-sets, which are to be expected in an industrial environment, data of up to 5\% perturbation in amplitude are produced by artificial inaccuracies during the measurement process, which offers a pool of about 300 data-sets. Further investigations show that some very small damages (e.g. very small flaws) are better observable, if more than one spectra of different measurement points is taken into account. 


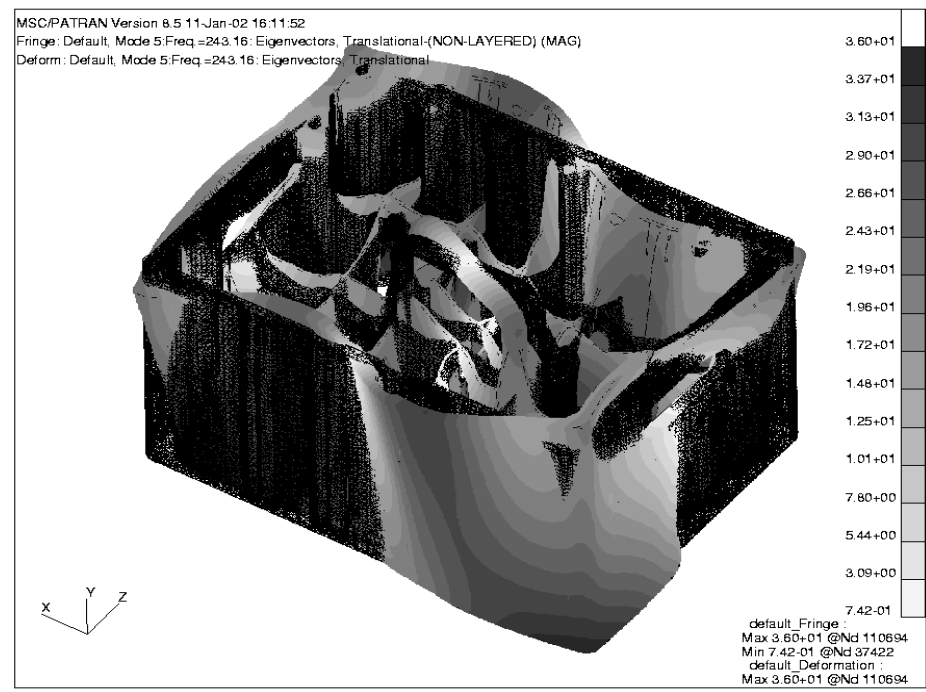

Fig. 3. Fifth mode shape at $243 \mathrm{~Hz}$

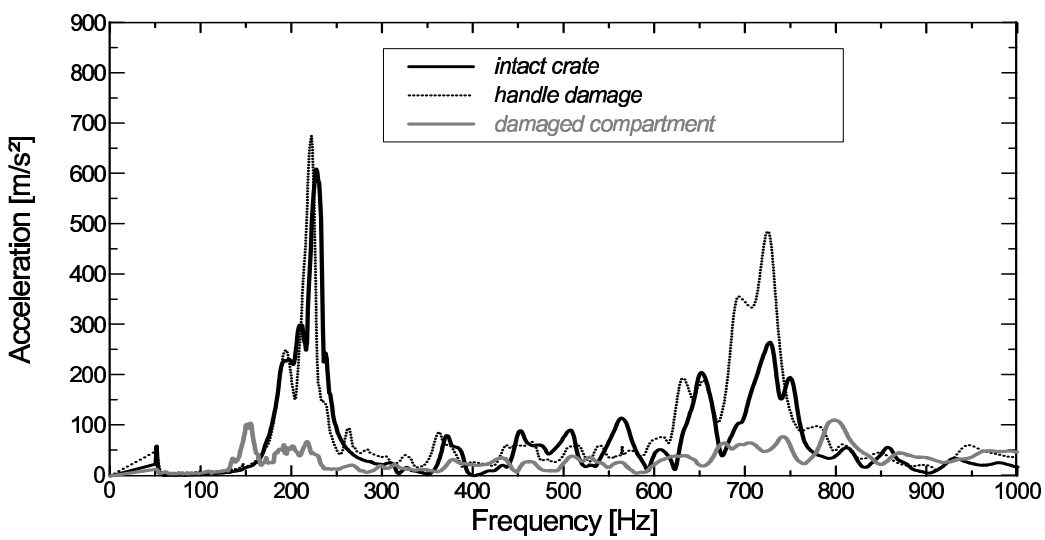

Fig. 4. Examples of vibration spectra of two different damages (handle reference point)

In order to limit the degrees of freedom of the ANN, the number of input nodes has been limited to 10 . This requires a reduction of the spectrum data-sets by a factor of hundred. Different methods have been applied. Splitting the spectrum into ten parts and integration of each part leads to an appropriate data reduction. Scaling, normalising, shifting or calculating of for example inertia moments did not improve the results. Figure 5 shows four different cases, where the input values of the nodes are displayed as bars. The data-reduction for both numerically and experimentally obtained spectra is carried out in the same way. 


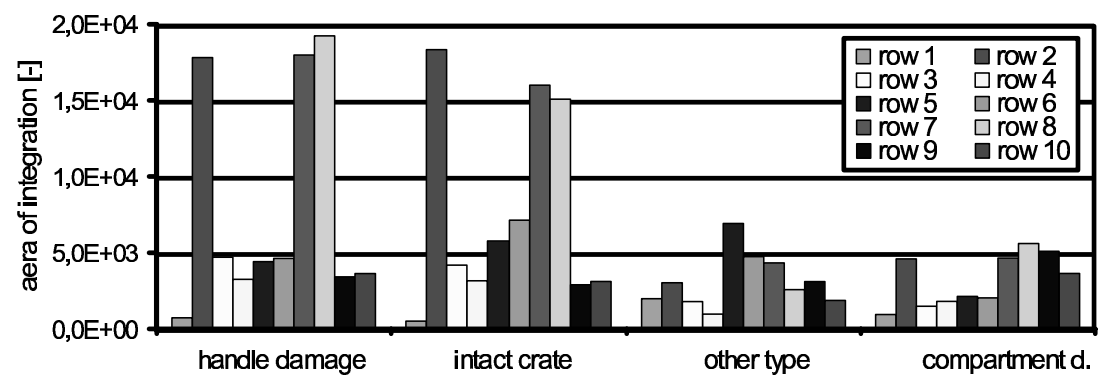

Fig. 5. Input data for ANN, bar plot of four different cases

\subsection{ANN trained by experimental data-sets}

In a first step, the ANN is processing exclusively experimental data in order to prove the feasibility of the pattern-recognition-system. The configuration of the network has been modified in order to analyse the sensitivity of the net topology. In all tests a MLP is used, trained by RPROP algorithm. Using more than 10 input nodes and more than 5 hidden nodes did not lead to any improvement in the prediction. Using less nodes resulted in worse predictions. If more output nodes are used, additional features of damages can be classified. For example a detection of different damage classes (e.g. "broken handle" or "broken compartment") is then possible. Damages that can not be identified, are automatically put into the class of "unknown" damages. The $10 \times 5 \times 2$ network yields a prediction of the defined output values (" 1 " and " 0 ") with an accuracy of about $5 \%$ for all the data-sets.

\subsection{ANN trained by a combination of numerical and experimental data-sets}

The frequency response obtained by numerical simulation has to agree with the experimental data in order to make sure that both methods can be applied. Experimental and numerical data of a frequency response analysis obtained by the use of 4 node and 10 node tetrahedral elements for an intact crate are shown in figure 6.

It can be stated, that the observable main characteristics of the curves are similar. In addition, there are two regions of interest where the numerical data are similar to those of the experimental response (around $200 \mathrm{~Hz}$ and above $500 \mathrm{~Hz}$ ). It is obvious that the simulation by the 10 node element model is closer to the experimental spectra than the 4 node element model. Further improvement of the agreement of the numeric simulation with the experiment is required. Nevertheless, simulations of damaged crates have been carried out in order to investigate their accuracy. As in the measured spectra (see figure 4) it can be stated that damages can be identified by comparison of the spectra. To estimate their quality, the comparison of numerical and experimental data of a handle damage is presented in figure 7. 


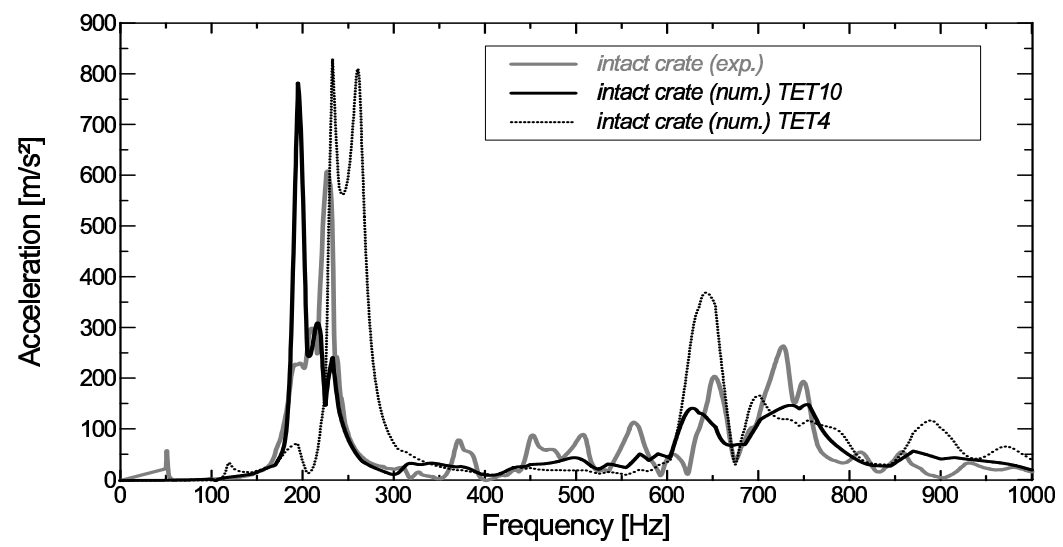

Fig. 6. Comparison of experimental and numerical spectra simulated by 4 node and 10 node tetrahedral elements (handle reference point)

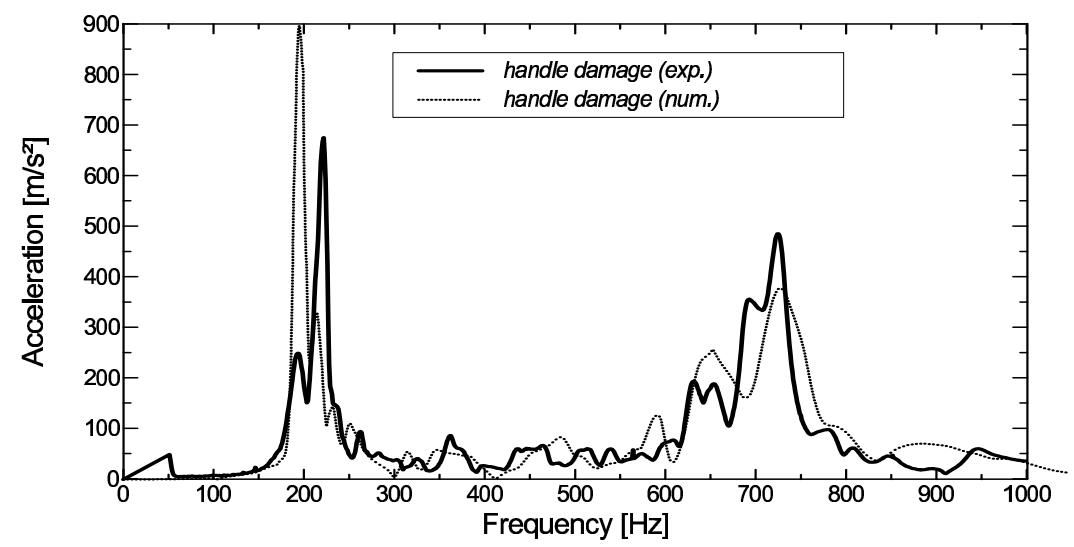

Fig. 7. Comparison of experimental and numerical handle damage (handle reference point)

The calculated response spectra as well as the experimental response spectra show net changes of peak size and location in similar frequency ranges. The difference between model and measurement of the undamaged crate in the example is, as [11] reported for a cracked beam, in the order of the change that the damage on the handle causes in measurement as well as in the simulation data. Because of this overlapping, it is important to be aware of the problem of false indication of damage in the two categories "false-positive" (indication of damage when none is present) and "falsenegative" (no indication of damage when damage is present) [19]. However, other damages and other crate models cause serious changes in the spectra so that these cases differ significantly, which allows their classification. 
At the moment, the objective of a training without experimental data-sets of these complex model is not yet met. Due to the existing difference between the numerical data-sets and the experimental data-sets, at the moment only an additional support use of the numerical data-sets is possible. Nevertheless, these first results point out the feasibility of network-training with simulated data. As the quality of the numerical simulation will be improved and data-reduction will be refined the classification is expected to become better.

\section{Conclusion and Perspectives}

In the present contribution, a pattern recognition method has been developed in order to detect damages on crates of beverages. Therefore, vibration response data of both damaged and intact crates have been recorded. Net differences can be observed with respect to the intact crate in all analysed damage cases. As the attempt is to use as less as possible "expert know-how", an ANN has been trained such that the detection of damaged crates is possible in all cases. Finite-element-simulation is carried out to analyse the mode shapes and in order to obtain data for the ANN-training. An updating of the FE-model meshing with ten node tetrahedral elements causes an enhancement of the agreement to measured data. While in the FE-data, damages can also be recognised easily, the agreement between experimental data and numerical data is not yet satisfying with regard to the little changes of some damages. Moreover the FE-data are exploited to get insight into the basic vibration behaviour. This is important to determine locations where sensors can be placed in order to obtain damage-representative data. The data reduction by splitting the spectra in equal sized parts and integration of each part over the individual frequency range has been proved to be an appropriate method.

The next steps in the current project will consist of a further improvement of the agreement between numerical and experimental data. This might be obtained by an anisotropic behaviour of the sensor or the idealised material parameters in the numerical model. Further improvement in availability of data is expected from the use of more than one measurement point on a crate and more data-sets to assure the reliability of the prediction of the ANN.

As a long term objective ageing or micro damages should be recognised by the system. For application of the method in an industrial context a reduction of the cycle time is required. While at the moment the cycle time is about 20 seconds, it has to be reached a level of one to two seconds. This can be obtained, if the excitation can be modified such that a shock excitation replaces the sinusoidal sweep which represents the bottle-neck of the current procedure. Also alternative processes are possible for the measurement of vibration signals, for example using contactless measurement systems or directly the signals on gripper devices. 


\section{References}

1. Klein, F.: Leergutsortierung in der Getränketechnik, Getränketechnik, 8 (1992) 46-52

2. Hermans, L., Van der Auweraer, H.: Modal Testing and Analysis of Structures under Operational Conditions: Industrial Applications, Mechanical Systems and Signal Processing, 13(2) (1999) 193-216

3. Doebling, S.W., Farrar, C.R., Prime, M.B.: A Summary Review of Vibration-Based Damage Identification Methods, The Shock and Vibration Digest, 30(2) (1998) 91-105

4. Salawu, O.S.: Detection of structural damage through changes in frequency: a review , Engineering Structures, 19(9) (1997) 718-723

5. Wang, Z., Lin, R.M., Lim, M.K.: Structural damage detection using measured FRF data, Computer Methods in Applied Mechanics and Engineering, 147 (1997) 187-197

6. Alampalli, S.: Effects of Testing, Analysis, Damage, and Environment on Modal Parameters, Mechanical Systems and Signal Processing 14(1) (2000) 63-74

7. Marwala, T., Hunt, H.E.M.: Fault Identification Using Finite Element Models and Neural Networks, Mechanical Systems and Signal Processing 13(3) (1999) 475-490

8. Masri, S.F., Nakamura, M., Chassiakos, A.G., Caughey, T.K.: Neural Network Approach to Detection of Changes in Structural Parameters, Journal of Engineering Mechanics, 122(4) (1996) 350-360

9. Elkordy, M.F., Chang, K.C., Lee, G.C.: Neural Networks Trained by Analytically Simulated Damage States, Journal of Computing in Civil Engineering, 7(2) (1993) 130-145

10. Kudva, J.N., Munir, N., Tan, P.W.: Damage Detection in Smart Structures Using Neural Networks and Finite-Element-Analyses, Smart Materials and Structures, 1 (1992) 108-112

11. Fritzen, C.-P., Jennewein, D.: Damage Detection Based on Model Updating Methods, Mechanical Systems and Signal Processing, 12(1) (1998) 163-186

12. Zacharias, J., Hartmann, Ch., Delgado, A.: Recognition of Damages on Crates of Beverages by an Artificial Neural Network, Conference-Proceedings of eunite 2001, Tenerife, Spain, (2001)

13. Reynier, M.: Sensors Location for Updating Problems, Mechanical Systems and Signal Processing, 13(2) (1999) 297-314

14. Entrekin, A.: Accuracy of MSC/NASTRAN First- and Second-Order Tetrahedral Elements in Solid Modeling for Stress Analysis, MSC Aerospace Users' Conference, (1999)

15. Sitton, G.: MSC/NASTRAN Basic Dynamic Analysis User's Guide, The McNealSchwendler Corporation, USA, (1997)

16. Rieg, F., Hackenschmidt, R.: Finite Elemente Analyse für Ingenieure, Hanser-Verlag, München, (2000)

17. Hagedorn, P., Otterbein, S.: Technische Schwingungslehre - Lineare Schwingungen diskreter mechanischer Systeme, Springer-Verlag, (1987)

18. Rafiq, M.Y., Bugmann, G., Easterbrook, D.J.: Neural Network Design for Engineering Applications, 79 (2001) 1541-1552

19. Fugate, M.L.: Vibration-Based Damage Detection Using Statistical Process Control, Mechanical Systems and Signal Processing, 15(4) (2001) 707-721

20. http://www.lfp.blm.tu-muenchen.de/pa/Software/knn.htm, 15.01.2002

21. Zell, A.: Simulation Neuronaler Netze, Addison-Wesley, Bonn, (1994) 